\title{
Effects of cisplatin and thiosulfate upon auditory brainstem responses of guinea pigs
}

\author{
W.C. Otto ${ }^{1}$, R.D. Brown ${ }^{2}$, L. Gage-White ${ }^{1}$, S. Kupetz ${ }^{2}$, M. Anniko ${ }^{4}$, J.E. Penny ${ }^{3}$ \\ and C.M. Henley ${ }^{5}$ \\ Departments of 'Otolaryngology ${ }^{2}$ Pharmacology and Therapeutics, ${ }^{3}$ Anatomy, Lousiana State University School of Medicine, \\ Shreveport, Lousiana, U.S.A., ${ }^{4}$ Department of Otolaryngology-Head and Neck Surgery, Umea University, Umea, Sweden \\ and ${ }^{5}$ Kresge Hearing Research Institute, University of Michigan, Ann Arbor, Michigan, U.S.A.
}

(Received 21 December 1987; accepted 1 May 1988)

\begin{abstract}
Two side effects which limit the use of cisplatin in cancer chemotherapy are severe nephrotoxicity and ototoxicity. The concurrent administration of sodium thiosulfate with cisplatin reportedly protects from cisplatin nephrotoxicity, however. protection from ototoxicity has not been documented. The purpose of this study was to examine the efficacy of using thiosulfate to ameliorate the ototoxic effects of cisplatin. Toward this end, the effects of cisplatin alone, cisplatin administered concurrently with sodium thiosulfate (CIS/THIO), and sodium thiosulfate alone on the auditory brainstem response (ABR) of guinea pigs were compared. ABR waveforms, comparing latencies, amplitudes and response thresholds, were monitored before, immediately after, and 30 days post treatment. Sodium thiosulfate administered with cisplatin (CIS/THIO) consistently protected animals from hearing loss and surprisingly yielded significant increases in amplitude when compared to baseline and saline controls. However, ABRs of CIS/THIO animals returned toward baseline values after 30 days.
\end{abstract}

Cisplatin; Thiosulfate; Ototoxicity; Hearing loss; Auditory brainstem response; Guinea pigs

\section{Introduction}

Cis-Diamminedichloroplatinum (II) (cisplatin) has been demonstrated to have antineoplastic activity against genitourinary cancer, (Loehrer and Einhorn, 1984) head and neck cancer, (Jacobs et al., 1978) and some lung cancers (Loehrer and Einhorn, 1984; Klastersky, 1985). Among its reported toxic effects are nausea, vomiting, hyperuricemia, lcukopenia, thrombocytopenia, and nephrotoxicity (Kovach et al., 1973; Strauss et al., 1983; Loehrer and Einhorn, 1984). In addition, a high rate of peripheral neuropathy (Thompson et al., 1984; Von Hoff et al., 1979) and hearing loss (Kovach et al., 1973; Helson et al., 1978; McHaney et al., 1983; Strauss et al., 1983) have been

Correspondence to: Walter C. Otto, Department of Otolaryngology, Louisiana State University School of Medicine, P.O. Box 33932, Shreveport LA 71130, U.S.A. reported. This hearing loss has been described as being directly related to cumulative dose, primarily affecting the basal turns of the cochlea (Helson et al., 1978; Reddel et al., 1982). Animal ototoxicity studies reveal that cisplatin mainly damages outer hair cells (Nakai et al., 1982; Stadnicki et al., 1975; Fleischman et al., 1975).

Several treatment regimens have been used to minimize the nephrotoxicity of cisplatin therapy (Higby et al., 1973; Comis, 1980; Kurebe et al., 1985). One of the more promising regimens, used for treating various types of neoplasms, employs two-route chemotherapy, (Helson et al,, 1978) using concurrent administration of cisplatin and sodium thiosulfate. Various combinations of two-route chemotherapy (i.e. i.p. cisplatin/i.v. thiosulfate, (Howell et al., 1983) i.a. cisplatin/i.v. thiosulfate, (Uozumi et al., 1983) i.v. cisplatin/i.v. sodium thiosulfate via separate sites (Pleifle et al., 1985) reportedly protect against nephrotoxicity 
and, in some cases, result in enhancement of the anticancer effect (Howell et al., 1983; Uozumi et al., 1983). Thiosulfate has been described as a free radical scavenger. When it interacts with cisplatin it apparently binds covalently to produce an inactive compound. (Howell et al., 1982).

The purpose of this study was to compare the effects of cisplatin alone, cisplatin administered concurrently with sodium thiosulfate, and sodium thiosulfate alone upon the auditory brainstem response (ABR) of guinea pigs. Latencies, amplitudes, and thresholds of ABR wave forms were compared. The cochleas and brains of each animal were collected and will be the subject of later reports.

\section{Methods}

\section{Animals}

Fifty-six female albino guinea pigs (425-690 g) were used in these experiments. There was no evidence of middle ear pathology and all animals had well-formed auditory brainstem responses and normal thresholds before treatment began (i.e., at baseline).

\section{Auditory evoked potentials}

ABRs were recorded before drug treatment, two days post treatment and 30 days or more post drug treatment. Prior to ABR testing, each guinea pig was sedated with a $0.39 \mathrm{ml} / \mathrm{kg}$ mixture of Ketamine and Xylazine $(3 \mathrm{mg} / \mathrm{ml}$ Ketalar to 1 $\mathrm{mg} / \mathrm{ml}$ Rompun). Animals were placed in a sound suite (Ray Proof Corp., Model AS216) during all recording sessions. Subdermal electrodes were inserted at the vertex (active electrode), right and left mastoids (ipsilateral and contralateral references) and right elbow (ground). Stimuli were rarefaction clicks generated by electrical square waves of $50 \mu \mathrm{s}$ presented to the right ear via an insert earphone (Etymotic Research, model ER-2) at rates of 27.1 and 77.1 clicks per s. Ipsilateral and contralateral responses were averaged using a Nicolet CA-1000 signal averager, high pass filtering at $30 \mathrm{~Hz}$ and low pass filtering at $3 \mathrm{kHz}$. Analysis time was $10 \mathrm{~ms}$ and sensitivity (artifact rejection) was set at $+/-25 \mu \mathrm{v}$. Using a repetition rate of 27.1 clicks per $s, 500$ responses were aver- aged at $10 \mathrm{~dB}$ decrements from $91 \mathrm{~dB}$ peak sound pressure level (SPL) down to visual detection threshold. The repetition rate was then increased to 77.1 per $s$ and responses were acquired at $81 \mathrm{~dB}$ peak SPL. Each averaged response was repeated to insure data reliability.

\section{Treatment}

Guinea pigs were divided into 5 groups. Group 1 (CIS-8) contained 17 guinea pigs which were injected with i.m. cisplatin $(1.5 \mathrm{mg} / \mathrm{kg} /$ day $)$ for 8 days. Group 2 (CIS/THIO) contained 11 guinea pigs which were injected with the same i.m. dose of cisplatin as group 1 but also given a concurrent dose of sodium thiosulfate, $1600 \mathrm{mg} / \mathrm{kg} /$ day i.p. for 8 days. Group 3 contained 10 guinea pigs which were injected i.m. with normal saline (1.5 $\mathrm{mg} / \mathrm{kg} /$ day) for 8 days. Group 4 (THIO) contained 6 animals which were injected i.p. with sodium thiosulfate $(1600 \mathrm{mg} / \mathrm{kg} /$ day $)$ for 8 days. Group 5 (CIS-5) contained 12 guinea pigs which were injected i.m. with cisplatin $(1.5 \mathrm{mg} / \mathrm{kg} /$ day $)$ for 5 days. Table I presents mean weights of each group.

\section{Statistical analysis}

Statistical analyses were performed using paired and unpaired, one-tailed Student's $t$ tests and analysis of variance in combination with the Tukey ' $a$ ' studentized range statistic which tests for highly significant differences. (Dixon and Massey, 1969; Winer, 1971). The level of significance accepted was alpha $=0.05$. However, attention was given to differences where $P<0.01$ using the Tukey 'a' test in order to be more confident of differences among the various means.

A series of one-way analyses of variance and studentized range statistics were performed on all ABR data. Between-group comparison of latency and amplitude on three different days for each of the 5 experimental groups, at 9 different intensities generated 540 between-group tests. Withingroup comparisons of the same data parameters resulted in an additional 270 tests. Presentation of such a mass of data in tabular form is prohibitive; therefore, only representative data at selected in. tensities are presented. 


\section{Results}

\section{Non-auditory findings}

Administration of $1.5 \mathrm{mg} / \mathrm{kg} /$ day doses of cisplatin for 8 days (CIS-8) resulted in overt signs of severe toxicity. All CIS-8 animals suffered weight loss, diarrhea, and loss of hair. Analysis of variance and studentized range tests revealed a significant decrease in the weight of CIS- 8 animals (Table I). All CIS-8 animals survived for 11 days, but by day 30 , five animals had died $(29 \%$ mortality 5 of 17).

All guinea pigs were weighed at regular intervals throughout the treatment period. Table I presents weight gain or loss for each group comparing the treatment period (days 1-8) with the total time period (days $1-30+$ ). While the saline control group and the CIS-5 animals steadily gained weight throughout, the CIS- 8 and CIS/THIO-8 animals steadily lost weight throughout the treatment and post treatment periods. According to the Tukey ' $a$ ' test, both the CIS- 8 and CIS/THIO-8 animals had significant weight loss relative to saline treated controls. Weight and hair

\section{TABLE I}

MEAN WEIGHT AT BASELINE AND MEAN WEIGHT LOSS/GAIN FOR EXPERIMENTAL GROUPS

\begin{tabular}{|c|c|c|c|}
\hline $\begin{array}{l}\text { Experi- } \\
\text { mental } \\
\text { group }\end{array}$ & $\begin{array}{l}\text { Mean } \\
\text { baseline } \\
\text { weight } \\
(N)\end{array}$ & $\begin{array}{l}\text { Mean weight } \\
\text { change during } \\
\text { treatment } \pm \text { S.E. } \\
(N)\end{array}$ & $\begin{array}{l}\text { Mean weight } \\
\text { change over } 30 \\
\text { days } \pm \text { S.E. } \\
(N)\end{array}$ \\
\hline CIS-8 & $\begin{array}{l}598.9 \mathrm{~g} \\
(17)\end{array}$ & $\begin{array}{l}-34.6 \pm 12.1 \mathrm{~g} * * \\
(17)\end{array}$ & $\begin{array}{l}-62.0 \pm 22.5 \mathrm{~g} * * \\
\text { (12) }\end{array}$ \\
\hline $\begin{array}{l}\text { CIS / } \\
\text { THIO-8 }\end{array}$ & $\begin{array}{l}593.8 \mathrm{~g} \\
(11)\end{array}$ & $\begin{array}{l}-11.1 \pm 9.0 \mathrm{~g} * * \\
(11)\end{array}$ & $\begin{array}{l}-20.6 \pm 13.5 \mathrm{~g} * \text { * } \\
(10)\end{array}$ \\
\hline SALINE & $\begin{array}{l}537.4 \mathrm{~g} \\
(10)\end{array}$ & $\begin{array}{l}48.6 \pm 4.1 \mathrm{~g} \\
(10)\end{array}$ & $\begin{array}{l}99.6 \pm 7.0 \mathrm{~g} \\
(10)\end{array}$ \\
\hline THIO & $\begin{array}{l}555.2 \mathrm{~g} \\
(6)\end{array}$ & $\begin{array}{l}-2.2 \pm 7.2 \mathrm{~g} \\
(6)\end{array}$ & (3) $11.7 \pm 19.2 \mathrm{~g}$ \\
\hline CIS-5 & $\begin{array}{l}604.5 \mathrm{~g} \\
(12)\end{array}$ & 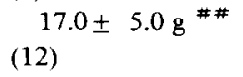 & $\begin{array}{l}25.2 \pm 6.9 \mathrm{~g} \\
(12)\end{array}$ \\
\hline
\end{tabular}

* = Significant difference from Saline Control $P<0.05$ (re. Tukey 'a')

** = Significant difference from Saline Control $P<0.01$ (re. Tukey ' $a$ ')

\# = Significant difference from CIS-8 $P<0.05$ (re. Tukey 'a')

\#\# = Significant difference from CIS-8 $P<0.01$ (re. Tukey 'a')
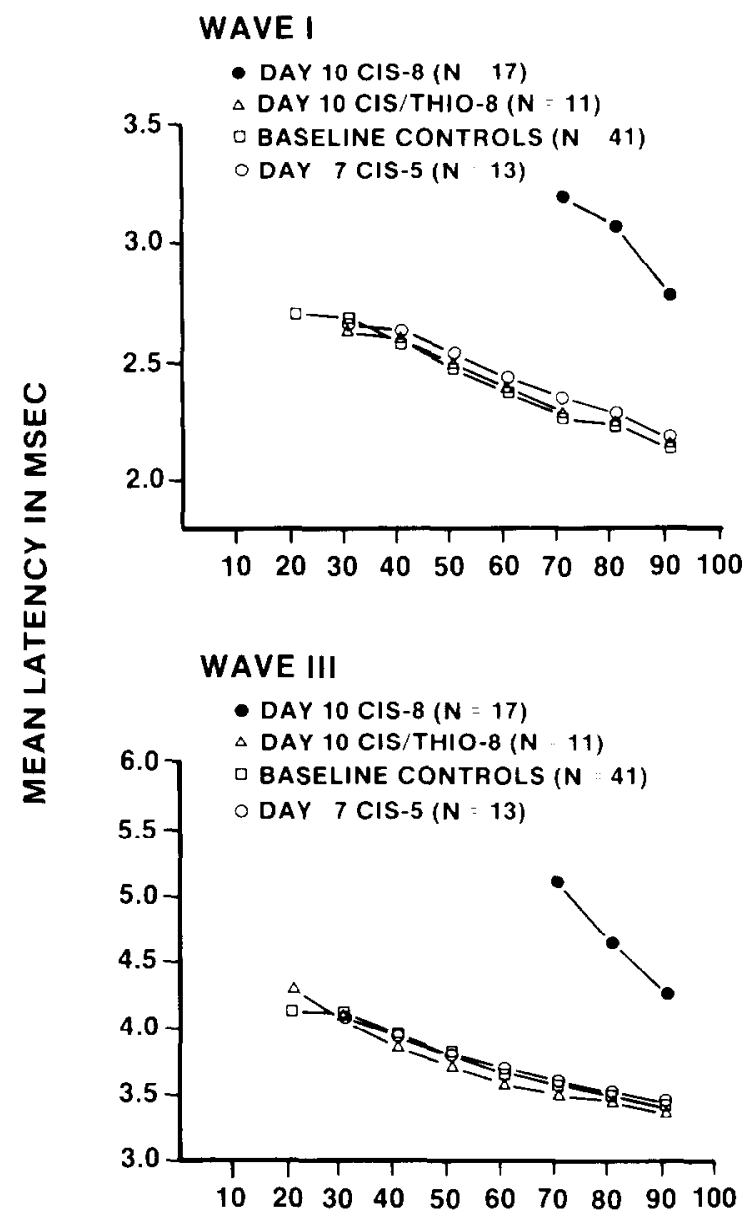

INTENSITY OF CLICK STIMULUS (DB PEAK SPL)

Fig. 1. Wave 1 and Wave III Latency-intensity functions for three experimental groups. Mean latencies before treatment (baseline) and post treatment are plotted for comparison. (CIS-8 = i.m. cisplatin treatment for 8 days: CIS/THIO-8 = i.m. cisplatin and i.p. sodium thiosulfate treatment for 8 days; CIS- $5=$ cisplatin treatment for 5 days; Baseline controls $=$ all experimental animals prior to treatment.)

loss was less apparent for CIS/THIO-8 animals and one death occurred following the course of treatment. The THIO animals had no weight gain until after the treatment period. Three of the six animals in this group did not survive to the end of the experiment. These deaths were attributed to the thiosulfate treatment since no other contributory factors could be identified. The only apparent systemic toxic effect for the CIS-5 group was a nonsignificant reduction in the rate of weight gain. 


\section{Auditory findings}

The CIS-8 group. All the CIS-8 animals displayed evidence of severe ototoxicity, suffering a $40 \mathrm{~dB}$ or greater threshold shift from baseline; 6 of 17 animals had no detectable ABR on day 10 . By day 30 , two of these six animals had died, but the four remaining animals had recovered sufficiently to measure latency at $91 \mathrm{~dB}$. At day 30 , the
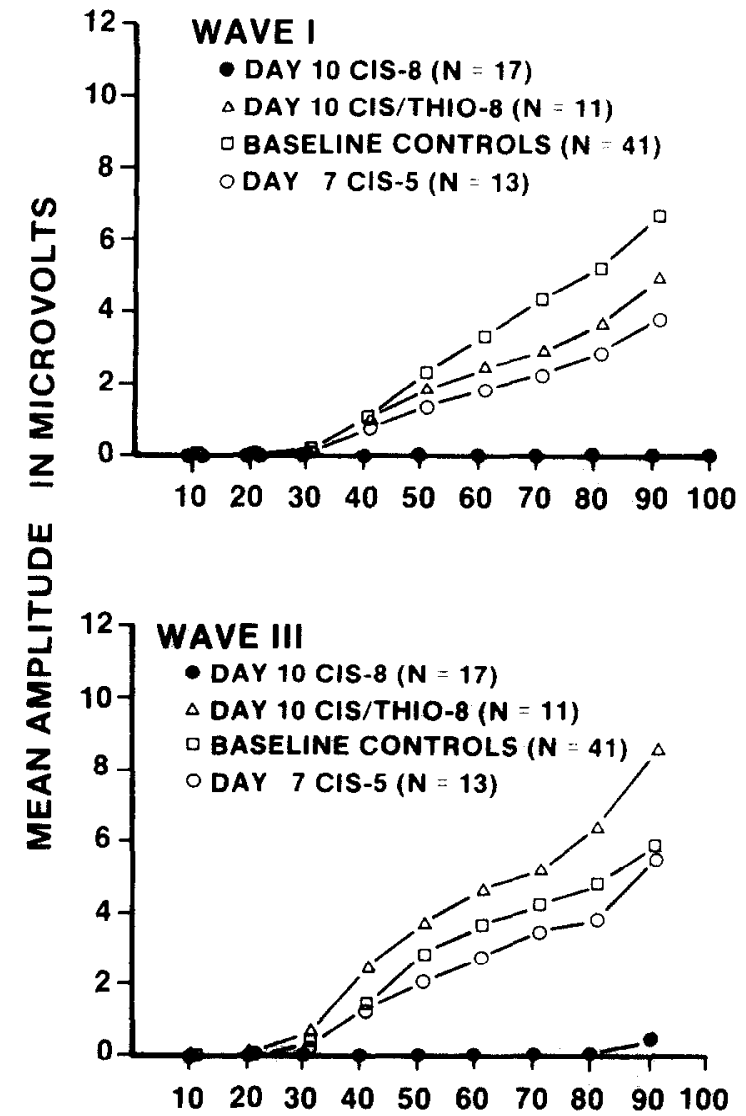

\section{INTENSITY OF CLICK STIMULUS} (DB PEAK SPL)

Fig. 2. Wave I and Wave III Amplitude-intensity functions for three selected experimental groups. Mean amplitudes before treatment (baseline) and post treatment are plotted for comparison (CIS-8 $=$ i.m. cisplatin treatment for 8 days; CIS/ THIO-8 $=$ i.m. cisplatin and i.p. sodium thiosulfate treatment for 8 days; CIS- 5 = cisplatin treatment for 5 days; BASELINE CONTROLS $=$ all experimental animals prior to treatment.) Note: Amplitudes for CIS-8 animals on day 10 at 71-91 dB are greater than $0.0 \mu \mathrm{V}$, but are undetectable from zero on this scale.
TABLE II

MEAN LATENCIES AND AMPLITUDES WITH S.E. FOR ABR WAVE III AT 91 dB PEAK SPL

\begin{tabular}{llc}
\hline & Latency in msec & $N$ \\
\hline CIS-8 (day 10) & $4.26 \pm 0.20$ & 11 \\
CIS/THIO (day 10) & $3.35 \pm 0.02$ & 11 \\
SALINE (day 10) & $3.44 \pm 0.05$ & 10 \\
THIO (day 10) & $3.36 \pm 0.04$ & 6 \\
CIS-5 (day 7) & $3.44 \pm 0.03$ & 11 \\
& Amplitude in $\mu \mathrm{V}$ & $N$ \\
\hline CIS-day (day 10) & $0.50 \pm 0.13$ & 17 \\
CIS/THIO-8 (day 10) & $8.56 \pm 0.92$ & 11 \\
SALINE (day 10) & $5.28 \pm 0.61$ & 10 \\
THIO (day 10) & $8.50 \pm 1.02$ & 6 \\
CIS-5 (day 7) & $5.47 \pm 0.82$ & 12 \\
\hline
\end{tabular}

other 11 survivors continued to manifest evidence of severe auditory impairment. Fig. 1 presents mean latencies by intensity for Waves I and III measured on day 10 . Only CIS-8 animals showed significant latency shifts. Fig. 2 presents mean amplitudes by intensity for Waves I and III measured on day 10.

In summary, the CIS- 8 group differed from all other groups (at the end of their respective treatments) on the following measures:

$\begin{array}{llll}\text { 1. Latency } & \text { Wave I } & \text { CIS-8 prolonged } & (P<0.01) \\ & \text { Wave III } & \text { CIS-8 prolonged } & (P<0.01) \\ \text { 2. Amplitude } & \text { Wave I } & \text { CIS-8 decreased } & (P<0.01) \\ & \text { Wave III } & \text { CIS-8 decreased } & (P<0.01)\end{array}$

On day 10 , CIS-8 animals also differed from their own baseline ABRs in all the categories listed previously. These highly significant differences persisted until the experiment was terminated (over 30 days). Table II presents mean latency and amplitude for Wave III of each experiment group at $91 \mathrm{~dB}$ peak SPL on day 10 (day 7 for CIS-5 animals).

The CIS/THIO-8 group. Auditory brainstem responses of the CIS/THIO-8 animals demonstrated no shifts in threshold; but subtle, significant changes in latency and amplitude did occur. Fig. 2 illustrates that wave III amplitudes for the CIS/THIO-8 animals at day 10 were consistently 
TABLE III

MEAN AMPLITUDE AND S.E. FOR WAVE I OF CIS/THIO-8 ANIMALS (91 dB PEAK SPL)

\begin{tabular}{lccc}
\hline & Day 0 & Day 10 & Day 30 \\
\hline Amplitude in $\mu \mathrm{V}$ & $8.25 \pm 0.39$ & $4.91 \pm 0.67$ & $5.28 \pm 0.59$ \\
Number of animals & 11 & 11 & 10 \\
\hline
\end{tabular}

greater than mean baseline values. Latencies of the CIS/THIO-8 animals were not significantly different from either the saline control or IHIO groups.

In summary, measurements for CIS/TIIIO animals at day 10 revealed only one significant difference from saline control animals:

$$
\begin{array}{lll}
\text { 1. Latency } & \text { Wave I } & \text { NS } \\
& \text { Wave III } & \text { NS } \\
\text { 2. Amplitude } & \text { Wave I } & \text { NS } \\
& \text { Wave III } & \text { CIS } / \text { THIO }>\text { Saline } \quad(P<0.05)
\end{array}
$$

Table III illustrates mean amplitudes plus or minus their standard errors for Wave $I$ of the CIS/THIO-8 animals when $91 \mathrm{~dB}$ stimuli were presented. Amplitudes at day 10 and at day 30 were significantly decreased $(P<0.01)$ relative to baseline. Decreases in amplitude of Wave I were also consistently significant at other intensities.

The THIO group. Amplitude and latencies for the THIO animals were not significantly different from saline controls or animals in the CIS/THIO-8 group, with one exception. Wave III amplitude was significantly greater than the saline group on day $10(P<0.05)$. As with CIS/THIO-8 group. these amplitudes returned to approximately baseline values by day 30 .

The CIS-5 group. No significant latency shifts occurred following CIS-5 treatment; neither within the group itself or in comparison with saline controls. Amplitudes of the CIS-5 animals decreased following treatment ( $P<0.05$ ), but only Wave III was significantly different from any other group on day 10 and day 30 :

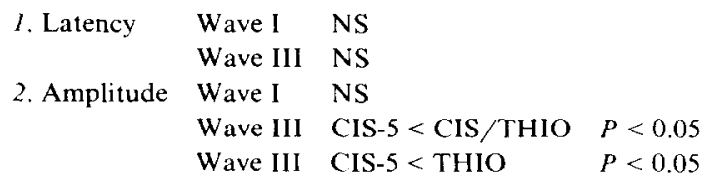

Fig. 3 illustrates the effect of CIS- 8 treatment regimen on the $A B R$ of a representative guinea pig of that group versus its own baseline ABR. Fig. 4 is a similar illustration of a guinea pig in the CIS/THIO-8 group. The degradation of the waveform in the CIS- 8 animal and the augmentation of Wave III amplitude in the CIS/THIO-8

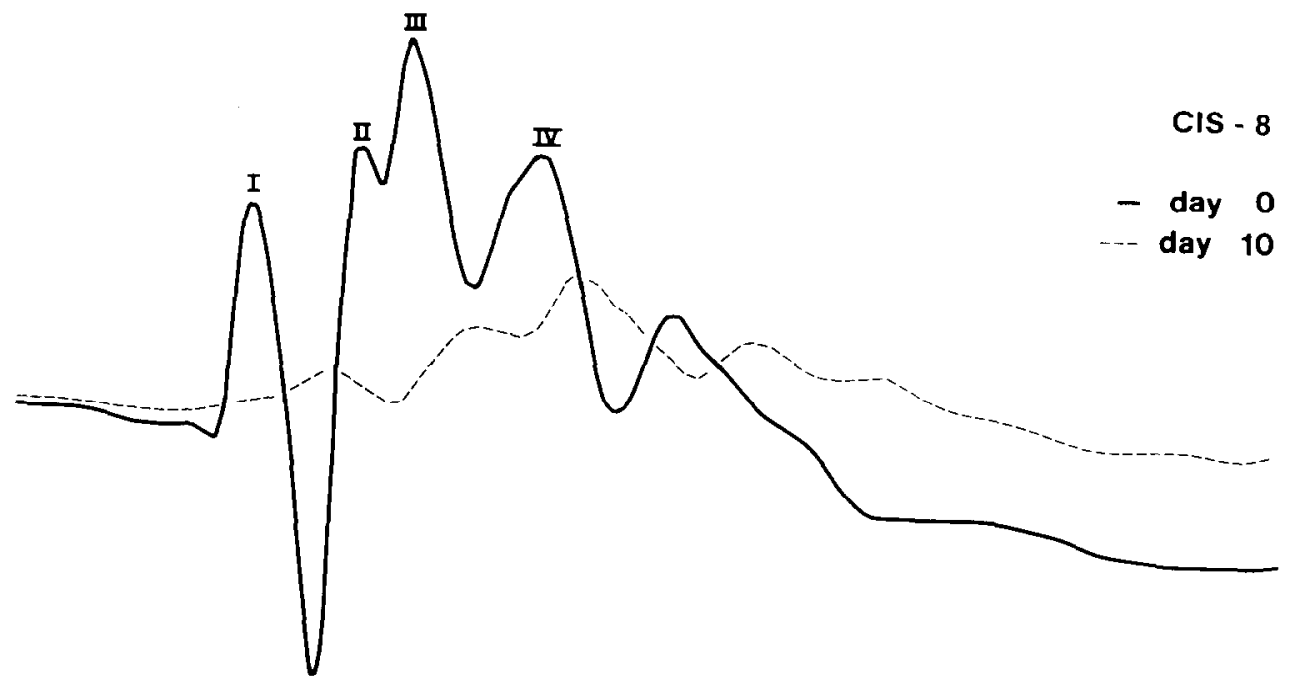

Fig. 3. Auditory brainstem response waveforms for a typical CIS-8 Guinea pig at baseline (prior to treatment) and on day 10 following i.m. cisplatin treatment $(1.5 \mathrm{mg} / \mathrm{kg} /$ day) for 8 days. Note diminished, distorted waveform after CIS-8 treatment. 


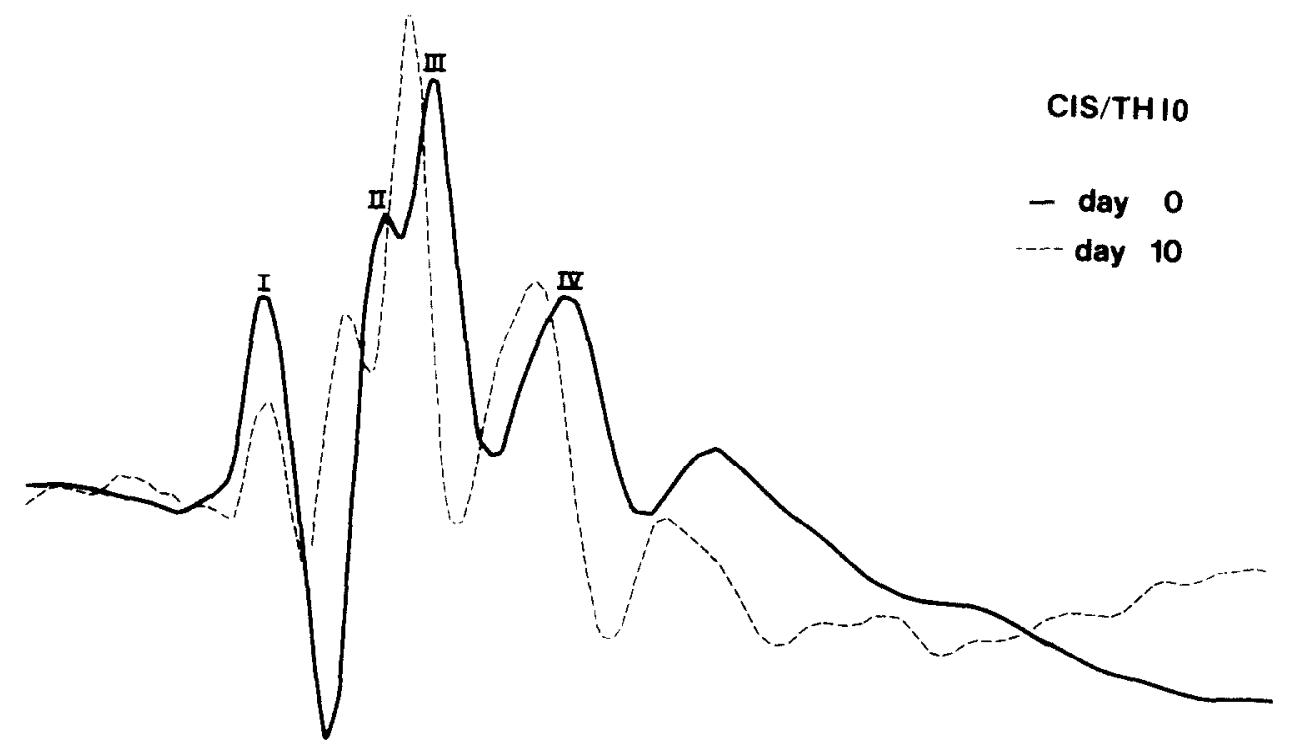

Fig. 4. Auditory brainstem response waveforms for a typical CIS/THIO-8 Guinea pig at baseline (prior to treatment) and on day 10 following i.m. cisplatin $(1.5 \mathrm{mg} / \mathrm{kg} /$ day) and i.p. sodium thiosulfate $(1600 \mathrm{mg} / \mathrm{kg} /$ day $)$ treatment for 8 days. Note increased amplitude and decreased latency of waves I and III following cis/THIO-8 treatment.

animal are characteristic of their respective treatment groups. These changes in waveform and amplitude are different from those in all other experimental groups and are statistically significant.

\section{Stimulus rate effect}

Responses were compared at two click rates $(27.1 / \mathrm{s}$ and $77.1 / \mathrm{s})$ at $81 \mathrm{~dB}$ peak SPL but results revealed no significant differences between groups in either latency or amplitude. Subjective evaluation of morphology did not reveal differences between groups.

\section{Discussion}

From these experiments we conclude that sodium thiosulfate protects the auditory system from the major ototoxic effects of cisplatin. In addition, the cisplatin/thiosulfate regimen appears to lessen other overt signs of systemic toxicity.

The significant increase in Wave III amplitude following CIS/THIO and THIO treatment provides an interesting topic for conjecture. Changes in this portion of the waveform indicate changes within the central auditory pathway. Perhaps this increase in amplitude is due to a release of normal efferent inhibition caused by the CIS/THIO combination or the thiosulfate alone. Perhaps there was a change in resting membrane potentials within the brainstem. This study was not designed to address that question. Since the wave III amplitude is normal on day 30 , the elevated amplitude is transient and therefore probably associated with an acute effect of the drugs. The authors are presently studying effects of thiosulfate alone upon the ABR.

ABR Wave I consistently diminished in amplitude following CIS/THIO treatment. Since wave I corresponds with the action potential of the VIIIth nerve, a decrease in amplitude indicates a decrease in function of the cochlea or the auditory nerve. The most parsimonious explanation of this finding is that cisplatin in the CIS/THIO treatment has a deleterious effect upon the cochlea, but far less than the same dose of cisplatin alone.

In light of the proven benefits derived from cisplatin as an anticancer agent, we are encouraged by our findings. Various two-route chemotherapy regimens combining cisplatin and sodium thiosulfate have been highly successful in eliminat- 
ing and reducing the size of tumors. Future investigations are needed to further explore applications of CIS/THIO therapy.

\section{References}

Comis, R.L. (1980) Cisplatin nephrotoxity: the effects of dose, schedule, and hydration scheme. In: A.W. Prestayko, St Crooke, S.K. Carger, (Eds.), Cisplatin: Current Status and New Developments, Academic Press, Inc., New York, pp. 485-494.

Dixon, W.J. and Masscy, F.J. (1969) Introduction to Statistical Analysis. (3rd ed.) McGraw-Hill, New York.

Fleischman, R.W., Stadnicki, S.W., Ethier, M.E. and Schaeppi, U. (1975) Ototoxicity of cis-dichlorodiammineplatinum (11) in the guinea pig. Toxicol. Appl. Pharmacol. 33, 320-332.

Helson, L., Okonkwo, E., Anton, L. and Cvitkovic, E. (1978) Cis-platinum ototoxicity. Clin. Toxicol. 13, 469-478.

Higby, D.J., Wallace, H.J. and Holland, J.F. (1973) Cis-diamminedichloroplatin (NSC-119875): a phase I study. Cancer Chemother. Rep., 57, 459-463.

Howell, S.B., Pfeifle, C.E., Wung, W.E., Olshen, R.A., Lucas, W.E., Yon, J.L. and Green, M. (1982) Intraperitoneal cisplatin with systemic thiosulfate protection. Ann. Inter. Med. 97. 845-851.

Howell, S.B., Pfeifle, C.E., Wung, W.R. and Olshen, R.A. (1983) Intraperitoneal cis-diamminedichloroplatinum with systemic thiosulfate protection. Cancer Res. 43, 1426-1431.

Jacobs, C., Bertino, J.R. and Goffinet, D.R. (1978) Cis-platinum chemotherapy in head and neck cancers. Oto Rhino Laryngol. 86, 780-783.

Klastersky, J. (1985) VP-16 and cisplatin in the treatment in non-small-cell lung cancers. Sem. Oncology 17-20 (Suppl. 2).

Kovach, J.S., Moertel, C.G., Schutt, A.J., Reitemeier, R.G. and Hahn, R.G. (1973) Phase II study of cis-diamminedichloroplatinum (NSC-119875) in advanced carcinoma of the large bowel. Cancer Chemother. Rep. 57, 357-359.

Kurebe, M.. Nuzato, T., Sanda, M., Sasaki, H. and Hayasaka,
H. (1985) Preventive effect of fosfomycin on the renal toxicity of cisplatin. Jap. J. Antibiot. 68 .

Loehrer, Pj. and Einhhorn, L.H. (1984) Cisplatin. Ann. Intern. Med. 100, 704-713.

McHancy, V.A., Thibadoux, G., Haycs, F.A. and Green, A.A. (1983) Hearing loss in children receiving cisplatin chemotherapy. J. Pediat. 102, 314-317.

Nakai, Y., Konishi, K. Chang, K.C., Ohashi, K., Morisaki, N., Minowa, Y. and Morimoto, A. (1982) Ototoxicity of the anticancer drug cisplatin. Acta Otolaryngol. 93, 227-232.

Pfeifle, C.E., Howell, S.B., Felthouse, R.D., Woliver, T.B.S., Andrews, P.A., Markman, M. and Murphy, M.P. (1985) High dose cisplatin with sodium thiosulfate protection. J. Clin. Oncol. 3, 237-244.

Reddel, R.R., Kefford, R.E., Grant, J.M., Coates, A.S., Fox, R.M. and Tattersall, M.H.N. (1982) Ototoxicity in patients receiving cisplatin: importance of dose and method of drug administration. Cancer Treat. Rep. 66, 19-23.

Stadnicki, S.W., Fleischman, R.W.. Schaeppi, U. and Merriam. P. (1975) Cis-dichlorodiammineplatinum (II) (NSC119875): hearing loss and other toxic effects in rhesus monkeys. Cancer Chemother. Rep. 59, 467-479.

Strauss, M., Towfighi, J., Lord, S., Lipton, A., Harvey, H.A. and Brown, B. (1983) Cis-platinum ototoxicity: clinical experience and temporal bone histopathology. Laryngoscope 93, 1554-1559.

Thompson, S.W., Davis, L.E., Kornfeld, M., Hilgers, R.D. and Standefer, J.C. (1984) Cisplatin neuropathy. Cancer 54. 1269-1275.

Uozumi, J., Sagiyama, K., Aoki, K., Iwamoto, and Baba T (1983) Effectiveness of "two-route chemotherapy" using cisplatin and its antidote sodium thiosulfate, on lifespan of rats bearing metastatic liver tumors. Cancer Treat. Rep. 67. 1067-1074.

Von Hoff, D.D., Reichert, C.M., Cuneo, R., Reddick, R.. Gallagher, M. and Rozencweig, M. (1979) Demyelination of peripheral nerves associated with cis-diamminedichloroplatinum (11) therapy. AACR Abstr. 91.

Winer, B.J. (1971) Statistical Principles in Experimental Design. (2nd ed.) McGraw Hill, New York. 